\title{
Measurement of $\mathrm{D}$-meson production in $\mathrm{pp}, \mathrm{p}-\mathrm{Pb}$ and $\mathrm{Pb}-\mathrm{Pb}$ collisions with ALICE at the LHC
}

\author{
A. Rossi for the ALICE Collaboration \\ CERN, Switzerland \\ Received 18 April 2014; received in revised form 23 July 2014; accepted 15 September 2014
}

Available online 24 October 2014

\begin{abstract}
Heavy quarks, i.e. charm and beauty, are considered calibrated probes for the strongly interacting deconfined medium (Quark Gluon Plasma, QGP) formed in heavy-ion collisions. Produced in hard scattering processes in the initial stages of the collision, they interact with the medium, lose energy and, depending on the coupling strength to the medium, take part in the collective motion of the QCD matter. ALICE measured the production of $\mathrm{D}^{0}, \mathrm{D}^{*+}, \mathrm{D}^{+}$and $\mathrm{D}_{\mathrm{s}}^{+}$mesons at central rapidity in $\mathrm{pp}, \mathrm{p}-\mathrm{Pb}$ and $\mathrm{Pb}-\mathrm{Pb}$ collisions at the LHC. The study of the modification of the transverse momentum differential yields of charm particles in $\mathrm{Pb}-\mathrm{Pb}$ collisions with respect to $\mathrm{pp}$ collisions, quantified by the nuclear modification factor $\left(R_{\mathrm{AA}}\right)$, can unravel details of the energy loss mechanism, such as its dependence on the quark mass and on the path length the parton travels through the medium. A similar comparison between $\mathrm{pp}$ and $\mathrm{p}-\mathrm{Pb}$ collision data $\left(R_{\mathrm{pPb}}\right)$ is fundamental to disentangle effects related to the presence of the hot medium from cold nuclear matter effects. The degree of thermalization and coupling to the medium is investigated in semi-peripheral $\mathrm{Pb}-\mathrm{Pb}$ collisions by measuring the elliptic flow coefficient $\left(v_{2}\right)$ at low $p_{\mathrm{T}}$. At high $p_{\mathrm{T}}, v_{2}$ is sensitive to the path-length dependence of the energy loss. Results on the transverse momentum and centrality dependence of the D-meson elliptic flow and $R_{\mathrm{AA}}$ will be presented. The comparison with the $R_{\mathrm{AA}}$ of non-prompt $\mathrm{J} / \psi$ from B-meson decays measured with CMS will be discussed. The preliminary results on D-meson $R_{\mathrm{pPb}}$ and the dependence of $\mathrm{D}$-meson yields on rapidity in $\mathrm{p}-\mathrm{Pb}$ collisions will be shown. As an outlook, the analysis and the preliminary results on the azimuthal correlations of D-mesons and charged hadrons in pp collisions will be described.
\end{abstract}

(c) 2014 CERN. Published by Elsevier B.V. All rights reserved.

Keywords: QGP; Charm; Heavy flavour; ALICE; Energy loss; Elliptic flow

E-mail address: andrea.rossi@cern.ch (A. Rossi). 


\section{Introduction}

The comparison of open heavy-flavour hadron production in proton-proton, proton- $\mathrm{Pb}$ and $\mathrm{Pb}-\mathrm{Pb}$ collisions at the LHC offers the opportunity to investigate the properties of the highdensity colour-deconfined state of strongly-interacting matter (Quark Gluon Plasma, QGP) that is expected to be produced in high-energy collisions of heavy nuclei [1]. Due to their large mass, charm and beauty quarks are created at the initial stage of the collision in hard-scattering processes with high virtuality $\left(Q^{2} \gtrsim 4 \mathrm{~m}_{c[b]}^{2}\right)$ involving partons of the incident nuclei. They interact with the medium and lose energy via both inelastic (medium-induced gluon radiation, or radiative energy loss) [2,3] and elastic (collisional energy loss) [4] processes. The loss of energy, sensitive to the medium energy density and size, is expected to depend on the quark mass and be smaller for heavy quarks than for light quarks and gluons for most of the mechanisms considered in theoretical models. In particular, the parton and mass dependence of radiative energy loss derives from the smaller colour coupling factor of quarks with respect to gluons, and from the 'dead-cone effect', which reduces small-angle gluon radiation for heavy quarks with moderate energy-over-mass values [5]. A sensitive observable is the nuclear modification factor, defined as $R_{\mathrm{AA}}\left(p_{\mathrm{T}}\right)=\frac{\mathrm{d} N_{\mathrm{AA}} / \mathrm{d} p_{\mathrm{T}}}{\left\langle T_{\mathrm{AA}}\right\rangle \sigma_{\mathrm{pp}} / \mathrm{d} p_{\mathrm{T}}}$, where $N_{\mathrm{AA}}$ is the yield measured in heavy-ion collisions, $\left\langle T_{\mathrm{AA}}\right\rangle$ is the average nuclear overlap function calculated with the Glauber model [7] in the considered centrality range, and $\sigma_{\mathrm{pp}}$ is the production cross section in pp collisions. In-medium energy loss determines a suppression, $R_{\mathrm{AA}}<1$, of hadrons at moderate-to-high transverse momentum $\left(p_{\mathrm{T}} \gtrsim 2 \mathrm{GeV} / c\right)$. The dependence of the energy loss on the parton nature (quark/gluon) and mass can be investigated by comparing the nuclear modification factors of hadrons with charm $\left(R_{\mathrm{AA}}^{\mathrm{D}}\right)$ and beauty $\left(R_{\mathrm{AA}}^{\mathrm{B}}\right)$ with that of pions $\left(R_{\mathrm{AA}}^{\pi}\right)$, mostly originating from gluon fragmentation at LHC energies. A mass ordering pattern $R_{\mathrm{AA}}^{\pi}\left(p_{\mathrm{T}}\right)<R_{\mathrm{AA}}^{\mathrm{D}}\left(p_{\mathrm{T}}\right)<R_{\mathrm{AA}}^{\mathrm{B}}\left(p_{\mathrm{T}}\right)$ has been predicted [5,6]. However, it is important to note that the comparison of heavy-flavour hadron and pion $R_{\mathrm{AA}}$ cannot be interpreted directly as a comparison of charm, beauty, and gluon energy losses, due to the different parton fragmentation functions and slope of the $p_{\mathrm{T}}$-differential cross sections (even in the absence of medium effects). Moreover, at low $p_{\mathrm{T}}$, a significant fraction of pions does not come from hard-scattering processes.

An $R_{\mathrm{AA}}$ value different from unity can also originate from initial and final state "cold-nuclear matter" effects, not related to the formation of a deconfined medium. At LHC energies, nuclear shadowing, which reduces the parton density for gluons carrying a nucleon momentum fraction $x$ below $10^{-2}$, is expected to be the most important for heavy-flavour production. A correct interpretation of heavy-ion results demands for the measurement of these effects via the analysis of $\mathrm{p}-\mathrm{Pb}$ data.

In heavy-ion collisions with non-zero impact parameter the interaction region exhibits an azimuthal anisotropy with respect to the reaction plane $\left(\Psi_{\mathrm{RP}}\right)$ defined by the impact parameter and the beam direction. Collective effects convert this geometrical anisotropy into an anisotropy in momentum space that is reflected in the final state hadron azimuthal distribution [8]. The effect, sensitive to the degree of thermalization of the system, can be evaluated by measuring the 2nd coefficient of the Fourier expansion of the particle azimuthal distribution, called elliptic flow $\left(v_{2}\right)$. The measurement of D-meson $v_{2}$ can provide, at low $p_{\mathrm{T}}$, fundamental information on the degree of thermalization of charm quarks in the medium. At high $p_{\mathrm{T}}$, a non-zero $v_{2}$ can originate from the path-length dependence of energy loss [9-14].

The measurement of both "hot" and "cold" nuclear effects requires the understanding of the production cross-sections of open heavy-flavour in pp collisions, used as a reference. The $p_{\mathrm{T}}$-differential production cross sections of $\mathrm{D}$ mesons is well described by fix-order pQCD cal- 

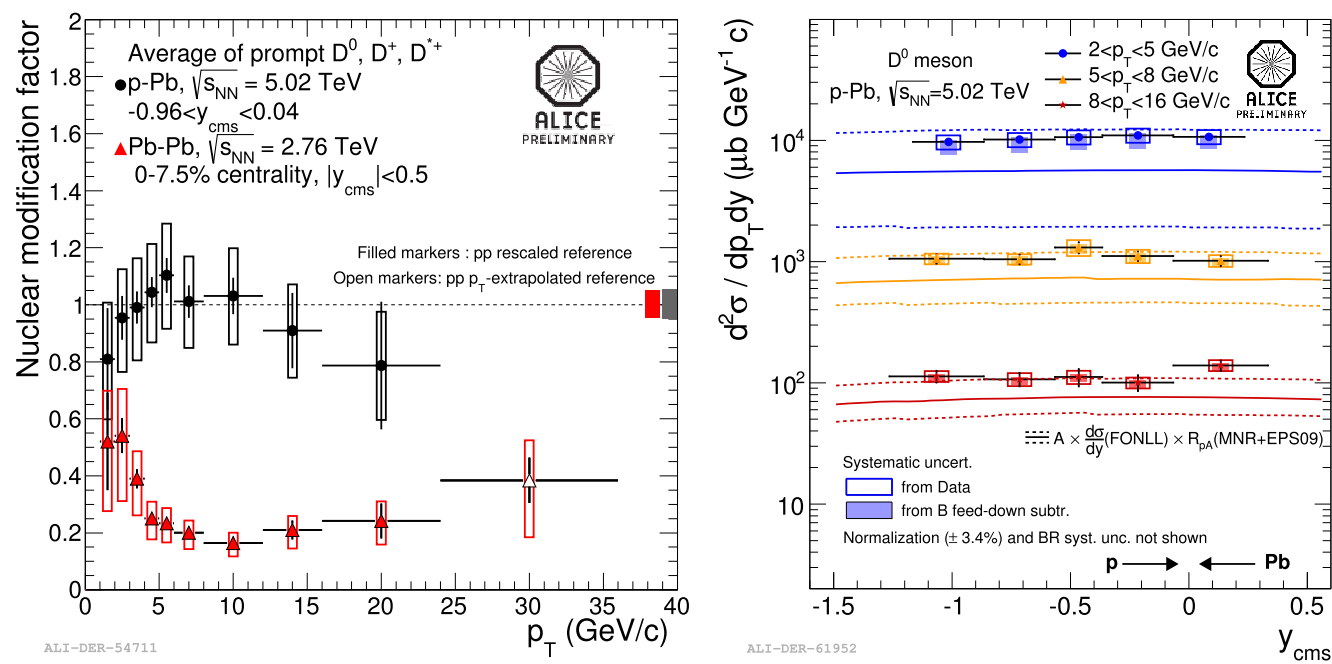

Fig. 1. Left panel: comparison of average $\mathrm{D}^{0}, \mathrm{D}^{*+}$ and $\mathrm{D}^{+}$nuclear modification factors measured in $\mathrm{p}-\mathrm{Pb}$ collisions and in the $0-7.5 \%$ most central $\mathrm{Pb}-\mathrm{Pb}$ collisions. Right panel: $p_{\mathrm{T}}, y$-differential cross-section for $\mathrm{D}^{0}$ production in $\mathrm{p}-\mathrm{Pb}$ collisions as a function of the rapidity in the centre of mass system for three different $p_{\mathrm{T}}$ ranges. The continuous and dashed lines represent expectations based on pQCD calculations including EPS09 parametrization of nuclear PDF [15, $24,22]$ (see text for more details).

culations relying on the collinear factorization approach, like FONLL [15], GM-VFNS [16], or the $k_{\mathrm{T}}$-factorization approach [17]. The study of the azimuthal angular correlations of D-meson and charged hadrons produced in the collisions offers the opportunity of investigating charm production in a more differential way and can serve as a test for Monte Carlo generators simulating the full kinematic of charm production processes and parton shower.

In these proceedings, the measurements of $\mathrm{D}$-meson production in $\mathrm{pp}, \mathrm{p}-\mathrm{Pb}$ and $\mathrm{Pb}-\mathrm{Pb}$ collisions performed by the ALICE Collaboration are presented.

\section{Analysis and results}

Open charm production is measured in the ALICE experiment via an invariant mass analysis of the decay channels $\mathrm{D}^{0} \rightarrow \mathrm{K}^{-} \pi^{+}(\mathrm{BR}=3.88 \%, c \tau=123 \mu \mathrm{m}), \mathrm{D}^{+} \rightarrow \mathrm{K}^{-} \pi^{+} \pi^{+}(\mathrm{BR}=$ $9.13 \%, c \tau=313 \mu \mathrm{m}), \mathrm{D}^{*+} \rightarrow \mathrm{D}^{0} \pi^{+}\left(\mathrm{BR}=68 \%\right.$, strong decay) and $\mathrm{D}_{\mathrm{s}}^{+} \rightarrow \phi \pi^{+} \rightarrow K^{+} K^{-} \pi^{+}$ $(\mathrm{BR}=2.28 \%, c \tau=149 \mu \mathrm{m})$ in pp collisions at $\sqrt{s}=2.76$ and $7 \mathrm{TeV}, \mathrm{p}-\mathrm{Pb}$ collisions at $\sqrt{s_{\mathrm{NN}}}=$ $5.02 \mathrm{TeV}$ and $\mathrm{Pb}-\mathrm{Pb}$ collisions at $\sqrt{s_{\mathrm{NN}}}=2.76 \mathrm{TeV}$. A similar analysis strategy was used in the different collision systems [18-21], based on exploiting the ALICE high track spatial resolution in the vicinity of the primary vertex of the collision $\left(\sim 60 \mu \mathrm{m}\right.$ at $\left.p_{\mathrm{T}}=1 \mathrm{GeV} / c\right)$, granted by the Inner Track System silicon detector (ITS), to identify secondary decay vertices displaced by few hundred $\mu \mathrm{m}$ from the interaction vertex. In order to further enhance the ratio between the D-meson signal and the large combinatorial background the measurements of the particle time-of-flight from the collision point to the Time Of Flight (TOF) detector and of the specific energy loss in the Time Projection Chamber (TPC) gas are used to identify kaons and pions. The pseudorapidity acceptance for tracks reconstructed in the ITS, TPC and TOF detectors is $|\eta|<0.9$. 

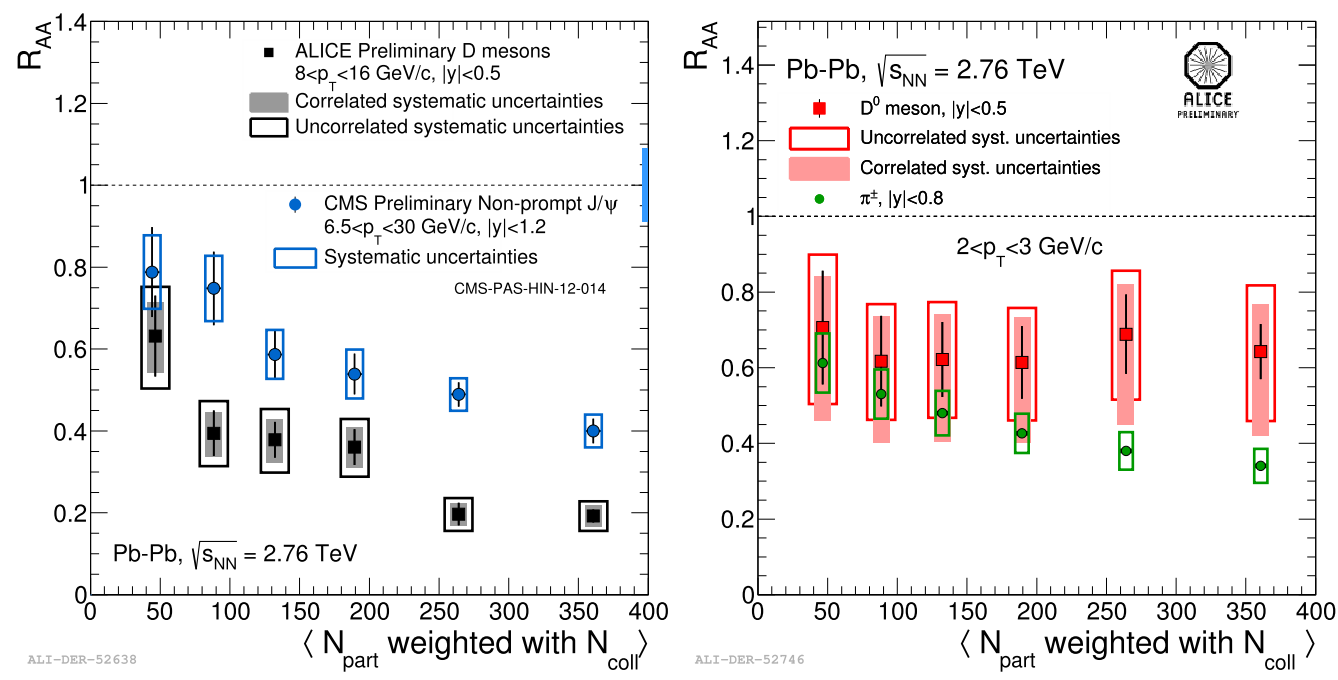

Fig. 2. Comparison of the centrality dependence of the nuclear modification factors of non-strange D mesons in $8<$ $p_{\mathrm{T}}<16 \mathrm{GeV} / c$ and $\mathrm{J} / \psi$ from B-meson decays measured with CMS [26] in $6<p_{\mathrm{T}}<30 \mathrm{GeV} / c$ (left panel) and of non-strange $\mathrm{D}$ mesons and charged pions in $2<p_{\mathrm{T}}<3 \mathrm{GeV} / c$ (right panel). The filled boxes represent the D-meson systematic uncertainties that are correlated in the different centrality intervals.

In the left panel of Fig. 1, the black points represent the ALICE preliminary average of prompt non-strange D-meson $R_{\mathrm{pPb}}$. As for the measurements performed in $\mathrm{pp}$ and $\mathrm{Pb}-\mathrm{Pb}[18,21]$, the contribution of $\mathrm{D}$ mesons coming from B-hadron decays is estimated and subtracted using the cross section of D mesons from B-meson decays calculated with FONLL [15], the reconstruction efficiency for prompt and secondary $\mathrm{D}$ mesons and using a range of hypotheses for the nuclear modification factor of feed-down D mesons $\left(R_{\mathrm{pPb}}^{\text {feed-down }}\right)$. In $\mathrm{p}-\mathrm{Pb}$ collisions $R_{\mathrm{pPb}}^{\text {feed-down }} / R_{\mathrm{pPb}}^{\mathrm{D}}=1$ is assumed and the range $0.9<R_{\mathrm{AA}}^{\text {feed-down }} / R_{\mathrm{AA}}^{\mathrm{D}}<1.3$ is spanned for assigning a systematic uncertainty. The range was chosen considering the predictions from calculations including initial state effects based on EPS09 nuclear PDF parametrizations [22] and from the model based on the Color Glass Condensate (CGC) framework described in [23]. $R_{\mathrm{pPb}}$ is compatible with unity in the range $1<p_{\mathrm{T}}<24 \mathrm{GeV} / c$. In the centre of mass system of the $\mathrm{p}-\mathrm{Pb}$ collisions, the measurement covers the rapidity range $-0.96<y<0.04$. Within the current statistical and systematic uncertainties, no evidence of a dependence of the production cross section on rapidity is observed within this window, as shown in the right panel of the same figure for $\mathrm{D}^{0}$ in $2<p_{\mathrm{T}}<5 \mathrm{GeV} / c$, $5<p_{\mathrm{T}}<8 \mathrm{GeV} / c$ and $8<p_{\mathrm{T}}<16 \mathrm{GeV} / c$. The measured $p_{\mathrm{T}}, y$-differential cross-section is compatible with predictions obtained by scaling the $y$-differential cross-section calculated with FONLL [15] by the $\mathrm{Pb}$ atomic mass number and by an $R_{\mathrm{pPb}}$ estimated, as a function of $p_{\mathrm{T}}$, on the basis of the MNR calculation [24] and EPS09 nuclear PDF parametrizations [22]. The latter $R_{\mathrm{pPb}}$ estimate describes well the measured $R_{\mathrm{pPb}}$ [25]. The comparison with the preliminary $R_{\mathrm{AA}}$ measured in the $7.5 \%$ most central $\mathrm{Pb}-\mathrm{Pb}$ collisions in $1<p_{\mathrm{T}}<36 \mathrm{GeV} / c$, shown in the left panel of Fig. 1, highlights that the suppression observed in $\mathrm{Pb}-\mathrm{Pb}$ collisions (about a factor 5 for $p_{\mathrm{T}} \sim 10 \mathrm{GeV} / c$ ) is predominantly induced by final state effects due to charm quark energy loss in the medium.

The preliminary comparison of the centrality dependence of the nuclear modification factors of non-strange $\mathrm{D}$ mesons and of $\mathrm{J} / \psi$ from B-meson decay measured with CMS [26], displayed 

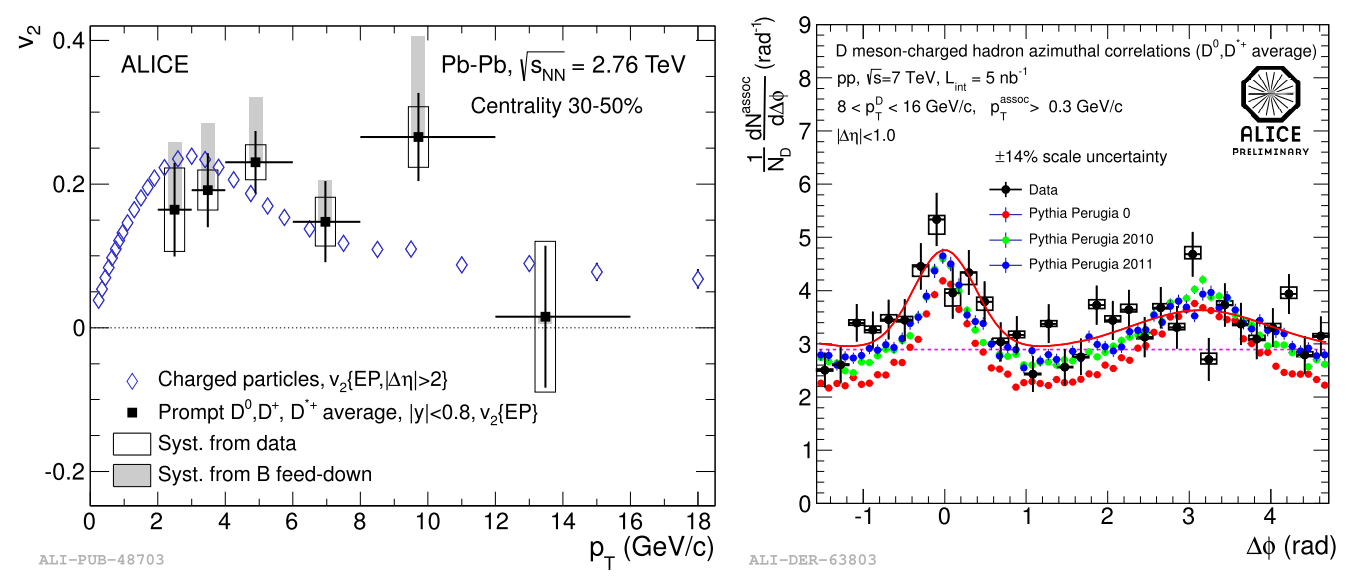

Fig. 3. Left panel (from [28]): comparison between $\mathrm{D}^{0}, \mathrm{D}^{*+}$ and $\mathrm{D}^{+}$average $v_{2}$ and charged hadron $v_{2}$ measured in $30-50 \%$ central $\mathrm{Pb}-\mathrm{Pb}$ collisions. Right panel: azimuthal correlations between $\mathrm{D}$ mesons (average of $\mathrm{D}^{0}$ and $\mathrm{D}^{*+}$ results) and charged hadrons in pp collisions at $\sqrt{s}=7 \mathrm{TeV}$, compared to expectations from simulations with the PYTHIA event generator $[32,33]$. The boxes represent the systematic uncertainty not correlated in $\Delta \phi$.

in the left panel of Fig. 2, represents an indication for a stronger suppression of charm than beauty in central $\mathrm{Pb}-\mathrm{Pb}$ collisions. The $8<p_{\mathrm{T}}<16 \mathrm{GeV} / c$ range was chosen for $\mathrm{D}$ mesons in order to have a similar kinematic range than that of $\mathrm{B}$ mesons decaying in a $\mathrm{J} / \psi$ in the measured $6.5<p_{\mathrm{T}}<30 \mathrm{GeV} / c$ range. For the subtraction of non-prompt $\mathrm{D}$ mesons from B-meson decays, on the basis of CMS results, $R_{\mathrm{AA}}^{\text {feed-down }} / R_{\mathrm{AA}}^{\mathrm{D}}=2$ was assumed as a central value and the range $1<R_{\mathrm{AA}}^{\text {feed-down }} / R_{\mathrm{AA}}^{\mathrm{D}}<3$ was considered for assigning the systematic uncertainty related to this hypothesis. As reported in [21,27], the D-meson $R_{\mathrm{AA}}$ is compatible within uncertainties with the charged-hadron $R_{\mathrm{AA}}$ for $p_{\mathrm{T}}>1 \mathrm{GeV} / c$ in central $\mathrm{Pb}-\mathrm{Pb}$ collisions. The same observation holds for more peripheral collisions, as also visible in the right panel of Fig. 2, where the centrality dependence of D-meson and charged-pion nuclear modification factors is shown for $2<p_{\mathrm{T}}<$ $3 \mathrm{GeV} / c$.

In the left panel of Fig. 3 the first measurement of D-meson elliptic flow in heavy-ion collisions is shown. The measurement, performed with ALICE in the 30-50\% centrality range [28], exploits the event plane method, in which the correlation of the particle azimuthal angle $(\varphi)$ to the reaction plane $\Psi_{\mathrm{RP}}$ is analyzed. The reaction plane is estimated via the event plane $\Psi_{2}$, which is obtained from the azimuthal distribution of a (sub-)sample of tracks in the event [29]. The measurement represents a $5 \sigma$ observation of $v_{2}>0$ in the range $2<p_{\mathrm{T}}<6 \mathrm{GeV} / c$, with an average of the measured values in this interval around 0.2 . A positive $v_{2}$ is also observed for $p_{\mathrm{T}}>6 \mathrm{GeV} / c$, which most likely originates from the path-length dependence of the partonic energy loss, although the large uncertainties do not allow a firm conclusion. The measured D-meson $v_{2}$ is comparable in magnitude to that of charged particles, which is dominated by light-flavour hadrons [30]. This suggests that low momentum charm quarks take part in the collective motion of the system.

As an outlook, in the right panel of Fig. 3, the first measurement of the azimuthal correlations between $\mathrm{D}$ mesons and charged particles in pp collisions at $\sqrt{s}=7 \mathrm{TeV}$ is shown (more details on the analysis technique can be found in [31]). The measurement, which is the average of $\mathrm{D}^{0}$ and $\mathrm{D}^{*+}$ correlations with charged particles, is compatible with the expectations obtained from the PYTHIA event generator [32] (tunes Perugia 0, Perugia 2010, and Perugia 2011 [33]), 
considering the large statistical and systematic uncertainties. The latter is dominated by the $14 \%$ uncertainty on the normalization. The larger statistics expected from upcoming runs at the LHC in 2014-2018, should allow for a precise measurement already in run 2 and the upgrade of the ALICE detector during the long shut down in 2018 [34] should give access to this observable also in $\mathrm{Pb}-\mathrm{Pb}$ collisions with run 3 data.

\section{References}

[1] P. Braun-Munzinger, J. Stachel, Nature 448 (2007) 302-309.

[2] M. Gyulassy, M. Plumer, Phys. Lett. B 243 (1990) 432.

[3] R. Baier, Y.L. Dokshitzer, A.H. Mueller, S. Peigne, D. Schiff, Nucl. Phys. B 484 (1997) 265.

[4] M.H. Thoma, M. Gyulassy, Nucl. Phys. B 351 (1991) 491;

E. Braaten, M.H. Thoma, Phys. Rev. D 44 (1991) 1298;

E. Braaten, M.H. Thoma, Phys. Rev. D 44 (1991) 2625.

[5] Y.L. Dokshitzer, D.E. Kharzeev, Phys. Lett. B 519 (2001) 199;

N. Armesto, C.A. Salgado, U.A. Wiedemann, Phys. Rev. D 69 (2004) 114003;

M. Djordjevic, M. Gyulassy, Nucl. Phys. A 733 (2004) 265;

B.-W. Zhang, E. Wang, X.-N. Wang, Phys. Rev. Lett. 93 (2004) 072301;

S. Wicks, W. Horowitz, M. Djordjevic, M. Gyulassy, Nucl. Phys. A 783 (2007) 493.

[6] N. Armesto, A. Dainese, C.A. Salgado, U.A. Wiedemann, Phys. Rev. D 71 (2005) 054027.

[7] J. Glauber, in: Lect. Theor. Phys. NY, vol. 1, 1959, p. 315;

M. Miller, et al., Annu. Rev. Nucl. Part. Sci. 57 (2007) 205.

[8] J.Y. Ollitrault, Phys. Rev. D 46 (1992) 229.

[9] O. Fochler, J. Uphoff, Z. Xu, C. Greiner, J. Phys. G 38 (2011) 124152.

[10] T. Lang, H. van Hees, J. Steinheimer, M. Bleicher, arXiv:1211.6912 [hep-ph];

T. Lang, H. van Hees, J. Steinheimer, Y.-P. Yan, M. Bleicher, arXiv:1212.0696 [hep-ph].

[11] P.B. Gossiaux, J. Aichelin, R. Bierkhandt, Phys. Rev. C 79 (2009) 044906;

P.B. Gossiaux, J. Aichelin, T. Gousset, V. Guiho, J. Phys. G 37 (2010) 094019.

[12] W.M. Alberico, et al., Eur. Phys. J. C 71 (2011) 1666;

W.M. Alberico, et al., J. Phys. G 38 (2011) 164144.

[13] W.A. Horowitz, M. Gyulassy, J. Phys. G 38 (2011) 124152.

[14] M. He, R.J. Fries, R. Rapp, Phys. Rev. C 86 (2012) 014903.

[15] M. Cacciari, M. Greco, P. Nason, JHEP 9805 (1998) 007.

[16] B.A. Kniehl, G. Kramer, I. Schienbein, H. Spiesberger, Eur. Phys. J. C 72 (2012) 2082.

[17] R. Maciula, M. Luszczak, A. Szczurek, arXiv:1207.6533 [hep-ph].

[18] B. Abelev, et al., ALICE Collaboration, JHEP 1201 (2012) 128.

[19] B. Abelev, et al., ALICE Collaboration, JHEP 1207 (2012) 191.

[20] B. Abelev, et al., ALICE Collaboration, Phys. Lett. B 718 (2012) 279-294.

[21] B. Abelev, et al., ALICE Collaboration, JHEP 1209 (2012) 112.

[22] K.J. Eskola, H. Paukkunen, C.A. Salgado, JHEP 0904 (2009) 065.

[23] H. Fujii, K. Watanabe, arXiv:1308.1258.

[24] M. Mangano, P. Nason, G. Ridolfi, Nucl. Phys. B 373 (1992) 295.

[25] G. Luparello, for the ALICE Collaboration, in: Proceedings of strangeness in Quark Matter 2013, arXiv:1310.1714.

[26] CMS Collaboration, 2012, CMS PAS HIN-12-014.

[27] A. Grelli, for the ALICE Collaboration, Nucl. Phys. A 904-905 (2013) 635c-638c.

[28] B. Abelev, et al., ALICE Collaboration, arXiv:1305.2707 [nucl-ex].

[29] A.M. Poskanzer, S. Voloshin, Phys. Rev. C 58 (1998) 1671, arXiv:nucl-ex/9805001.

[30] B. Abelev, et al., ALICE Collaboration, arXiv:1205.5761 [nucl-ex].

[31] F. Colamaria, for the ALICE Collaboration, in: Proceedings of SQM 2013, arXiv:1310.3621.

[32] T. Sjostrand, S. Mrenna, P. Skands, JHEP 05 (2006) 026.

[33] P.Z. Skands, Phys. Rev. D 82 (2010) 074018.

[34] ALICE Collaboration, Upgrade of the ALICE Experiment - Letter Of Intent, CERN-LHCC-2012-012/LHCC-I022 . 